University of Nebraska - Lincoln

DigitalCommons@University of Nebraska - Lincoln

Faculty Publications from the Department of Engineering Mechanics

Mechanical \& Materials Engineering,

Department of

6-29-2007

\title{
Adhesive contact in filaments
}

\author{
Xiang-Fa Wu \\ Department of Engineering Mechanics, University of Nebraska-Lincoln, xfwu@unlserve.unl.edu
}

Yuris A. Dzenis

Department of Engineering Mechanics, University of Nebraska-Lincoln, ydzenis@unl.edu

Follow this and additional works at: https://digitalcommons.unl.edu/engineeringmechanicsfacpub

Part of the Mechanical Engineering Commons

Wu, Xiang-Fa and Dzenis, Yuris A., "Adhesive contact in filaments" (2007). Faculty Publications from the Department of Engineering Mechanics. 19.

https://digitalcommons.unl.edu/engineeringmechanicsfacpub/19

This Article is brought to you for free and open access by the Mechanical \& Materials Engineering, Department of at DigitalCommons@University of Nebraska - Lincoln. It has been accepted for inclusion in Faculty Publications from the Department of Engineering Mechanics by an authorized administrator of DigitalCommons@University of Nebraska - Lincoln. 
Published in Journal of Physics D: Applied Physics 40 (2007), pp. 4276-4280; doi 10.1088/0022-3727/40/14/026

Copyright (C) 2007 IOP Publishing. Used by permission. http://stacks.iop.org/JPhysD/40/4276

Submitted May 4, 2007; revised May 23, 2007; published June 29, 2007.

\title{
Adhesive contact in filaments
}

\author{
Xiang-Fa Wu and Yuris A Dzenis \\ Department of Engineering Mechanics, Nebraska Center for Materials and Nanoscience, \\ University of Nebraska-Lincoln, Lincoln, NE 68588-0526, USA
}

Corresponding author - X.-F. Wu, email xfwu@unlserve.unl.edu

\begin{abstract}
This paper studies the elastic contact in filaments induced by surface adhesion, which plays an important role in the mechanical response of fibrous materials (e.g., fiber friction, sliding, compression hysteresis, etc.). During the process, a simple 3D elastic contact model was proposed. The filaments were assumed to be uniform, smooth elastic cylinders, and the adhesive force between filaments in contact was estimated according to Bradley's approach (Bradley 1932 Phil. Mag. 13 853) that relies on the filament configurations before deformation. Under the action of fiber surface adhesion, the elastic deformation and the size of the contact zone were determined in closed-form based on the DMT theory (Derjaguin et al. 1975 J. Colloid Interface Sci. 53 314). Effects of filament radius and orientation, surface energies and elasticity on the elastic deformation and the size of the contact zone were explored numerically. The model developed in this work can be used for the study of the mechanisms of filament contacts, friction, sliding and compression hysteresis in fibrous materials subjected to external loading.
\end{abstract}

\section{Introduction}

Fibrous materials have been used extensively in thermal and sound insulators, gas and fluid filters, chemical carriers, tissue templates and various paper products. As porous matter, fibrous material is made of thin fibers that have a very high surface area to volume ratio and tensile strength. As a matter of fact, the strength of a fibrous material relies on the fiber tensile and bonding strength and the specific fiber arrangement inside the fibrous material. Recently, ultrathin fibers produced by the electrospinning technique [1-3] further extend the applications of fibrous materials. So far, continuous ultrathin fibers with diameters ranging from a few microns down to tens of nanometers have been fabricated efficiently by electrospinning. With their continuity, high tensile strength, controllable surface morphology, and chemical structures, electrospun nanofibers have been utilized in enhancing the targeted functionalities in almost all fields where traditional fibrous materials have functioned as key structural and functional components and in other new frontiers (e.g. drug delivery, advanced nanofiber sensors, etc) due to their unique properties [4-8].

With the rapidly growing applications of advanced fibrous materials, their mechanical properties have evoked remarkable attention in recent decades. Special care has been taken for a long time to relate their effective stiffness and strength. To mention a few, van Wyk [9] was the first to develop a power-law pressure-volume relation for the compression of wool with $3 \mathrm{D}$ random orientation. In this model, the wool assembly was treated as a layered structure with fiber deflection between neighboring contacts. Furthermore, Cox [10] considered the effective moduli of dilute planar fiber networks (fiber composites) through averaging the stiffness contribution of individual fibers within a representative area element. Narter et al. [11] extended Cox's approach to 3D cases. Nevertheless, Cox's model only takes into account the average effect of individual fibers. Neither fiber-fiber bonds nor transverse fiber deflections were taken into account. Moreover, by refining the fiber contact and deflection assumptions, quite a few fiber network models have been proposed afterwards that were validated by experiments and numerical simulations [12-22]. Among these, Carnaby and Pan [12, 13] considered the effect of fiber friction and sliding on the mechanical response of fiber assemblies subjected to compression. They modeled the compression hysteresis of fiber assemblies based on the simple Columbus friction law. As a matter of fact, for fibers at small scale, surface adhesion may have an 


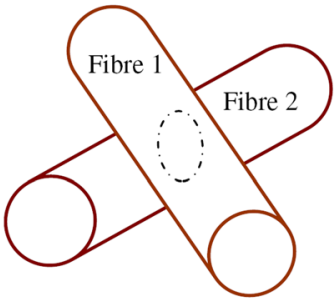

(a)

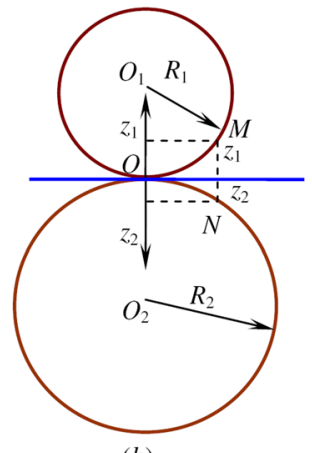

(b)
Figure 1. Cylindrical filaments in contact (a) contact zone between filaments in contact (b) coordinate systems.

appreciable effect on fiber contact, friction, sliding and resulting compression hysteresis, and it may even affect the fiber dynamics and collapse [23, 24]. However, to the authors' knowledge, no work has been reported yet in the literature regarding the surface effect on the mechanical properties of fibrous materials.

Therefore, in this work we initiate a study on the surface effect on fiber contacts in fibrous materials, which is expected to play an important role in the mechanical response of fibrous materials. In this modeling process, we propose a simple 3D elastic contact model based on the DMT theory [25], where the elastic contact of filaments is induced by surface adhesion between filaments in contact. Without loss of generality, the filaments are assumed to be uniform, smooth elastic cylinders. The adhesive force between filaments is estimated according to Bradley's approach [26]. The elastic deformation and the size of the contact zone are to be determined in closed form by Hertz contact theory [27-30]. Effects of filament radius and orientation, surface energies, and elasticity on the elastic deformation and the size of the contact zone are explored numerically. Consequently, the potential applications of the present model and relevant results are further addressed.

\section{Problem statement and solution}

Adhesive contact in filaments can be modeled as elastic contact between two elastic cylinders positioned at an arbitrary angle subjected to surface adhesion. It can be dealt with as the special case of the DMT theory [25] or the generalized Hertz contact theory [27-30]. Let us first introduce Hertz contact theory for the general contact of two elastic bodies and then use the relevant results to study adhesive contact in filaments. Consider the tangent plane at the point $O$ of two elastic bodies in contact as the $(x, y)$-plane, as illustrated in Figure 1. By ignoring the higher order terms, the surfaces near the contact point may be approximated by two polynomials:

$$
\begin{aligned}
& z_{1}=A_{1} x^{2}+A_{2} x y+A_{3} y^{2}, \\
& z_{2}=B_{1} x^{2}+B_{2} x y+B_{3} y^{2} .
\end{aligned}
$$

In the above $A_{i}(i=1,2,3)$ and $B_{i}(i=1,2,3)$ are the geometrical parameters describing the surfaces near the contact point above and below. As shown in Figure 1, the distance between two points at the two surfaces such as $M$ and $N$ is

$$
z_{1}+z_{2}=\left(A_{1}+B_{1}\right) x^{2}+\left(A_{2}+B_{2}\right) x y+\left(A_{3}+B_{3}\right) y^{2} .
$$

By properly rotating the $(x, y)$-coordinate system, the cross term $x y$ can be eliminated. Then, relation (2) can be recast into

$$
z_{1}+z_{2}=A x^{2}+B y^{2},
$$

where $A$ and $B$ are coefficients relating the principal curvatures of the surfaces in contact and the angle between the planes of principal curvatures of the two surfaces. By denoting $R_{1}$ and $R_{1}^{\prime}$ as the principal radii of curvature at the point of contact of the body above, $R_{2}$ and $R_{2}^{\prime}$ the body below, and $\varphi$ the angle between the two normal planes containing the curvatures $1 / R_{1}$ and $1 / R_{2}$, the coefficients $A$ and $B$ can be therefore determined:

$$
\begin{aligned}
A+B & =\frac{1}{2}\left(\frac{1}{R_{1}}+\frac{1}{R_{1}^{\prime}}+\frac{1}{R_{2}}+\frac{1}{R_{2}^{\prime}}\right), \\
B-A & =\frac{1}{2}\left[\left(\frac{1}{R_{1}}-\frac{1}{R_{1}^{\prime}}\right)^{2}+\left(\frac{1}{R_{2}}-\frac{1}{R_{2}^{\prime}}\right)^{2}\right. \\
+2 & \left.\left(\frac{1}{R_{1}}-\frac{1}{R_{1}^{\prime}}\right)\left(\frac{1}{R_{2}}-\frac{1}{R_{2}^{\prime}}\right) \cos 2 \varphi\right]^{1 / 2} .
\end{aligned}
$$

In the case of two uniform elastic cylindrical filaments in contact, relations in (4) are reduced to

$$
A=\frac{1-\cos \varphi}{2 R}, \quad B=\frac{1+\cos \varphi}{2 R},
$$

where $R$ is the radius of the filaments and $\varphi$ is the spatial angle between the filament axes. In relation (3), $A$ and $B$ have the same sign, therefore all points with the same mutual distance $z_{1}+z_{2}$ will be located on one ellipse. As a result, the contact surface of two cylindrical filaments will have an elliptical boundary.

Let a be the distance reduction between the two elastic bodies subjected to compression and $w_{1}$ and $w_{2}$ the displacements of the points on the surfaces in contact above and below, respectively. Then, for these points, the following relation holds such that

$$
w_{1}+w_{2}+z_{1}+z_{2}=\alpha
$$

or

$$
w_{1}+w_{2}=\alpha-A x^{2}-B y^{2} .
$$

The theory of elasticity [28] leads to the sum of the displacements $w_{1}$ and $w_{2}$ as

$$
w_{1}+w_{2}=\left(k_{1}+k_{2}\right) \int \frac{p}{r} \mathrm{~d} A,
$$

where $p \mathrm{~d} A$ is the pressure acting on an elemental area of the contact surface, $r$ is the distance from this elemental area to the contact center (see Figure $1(\mathrm{~b})$ ), and $k_{1}$ and $k_{2}$ are defined by

$$
k_{1}=\frac{1-v_{1}^{2}}{\pi E_{1}}, \quad k_{2}=\frac{1-v_{2}^{2}}{\pi E_{2}},
$$

where $E_{i}$ and $v_{i}(i=1,2)$ are Young's moduli and Poisson's ratios of the elastic bodies above and below, respectively. 
Table 1. $m$ and $N$ values for varying angle $\varphi$ [28].

\begin{tabular}{cccccccccccccccc}
\hline$\theta^{\circ}$ & $30^{\circ}$ & $35^{\circ}$ & $40^{\circ}$ & $45^{\circ}$ & $50^{\circ}$ & $55^{\circ}$ & $60^{\circ}$ & $65^{\circ}$ & $70^{\circ}$ & $75^{\circ}$ & $80^{\circ}$ & $85^{\circ}$ & $90^{\circ}$ \\
\hline$m$ & 2.731 & 2.397 & 2.136 & 1.926 & 1.754 & 1.611 & 1.486 & 1.378 & 1.284 & 1.202 & 1.128 & 1.061 & 1.000 \\
$N$ & 0.493 & 0.530 & 0.567 & 0.604 & 0.641 & 0.678 & 0.717 & 0.759 & 0.802 & 0.846 & 0.893 & 0.944 & 1.000 \\
\hline
\end{tabular}

The above integration must be performed over the entire surface in contact. Substituting (7) into (8) yields

$$
\left(k_{1}+k_{2}\right) \int \frac{p}{r} \mathrm{~d} A=\alpha-A x^{2}-B y^{2} .
$$

Hertz contact theory [27-29] indicates that pressure $p$ over the contact surface to satisfy the above equation is represented by the ordinates of a semi-ellipsoid that is spanned on the entire contact surface. The peak pressure $p_{0}$ is located at the center of the contact zone. By denoting $a$ and $b$ as the semi-axes of the ellipse of the contact zone, the peak pressure can be determined by

$$
P=\int p \mathrm{~d} A=\frac{2}{3} \pi a b p_{0},
$$

from which we have

$$
p_{0}=\frac{3}{2} \frac{P}{\pi a b} \text {. }
$$

In the above $P$ is the compressive force that is actually the resultant adhesive force between two filaments in contact. The peak pressure is 1.5 times the average pressure on the contact surface, and $a$ is related to the compressive force $P$ and the semi-axes of the ellipse $a$ and $b$ [29] such that

$$
\alpha=\frac{3 P\left(k_{1}+k_{2}\right)}{4} \int_{0}^{\infty} \frac{\mathrm{d} \xi}{\sqrt{\left(a^{2}+\xi\right)\left(b^{2}+\xi\right) \xi}},
$$

where the semi-axes $a$ and $b$ are determined as

$$
a=m \sqrt[3]{\frac{3 \pi}{4} \frac{P\left(k_{1}+k_{2}\right)}{A+B}}, \quad b=n \sqrt[3]{\frac{3 \pi}{4} \frac{P\left(k_{1}+k_{2}\right)}{A+B}} .
$$

In the above, coefficients $m$ and $n$ are numbers depending only on the ratio $(B-A)$ to $(A+B)$ as tabulated in the literature [28].

Now, let us consider the compressive force induced by surface adhesion between filaments in contact. As we know, the adhesive forces between filaments are distributed forces that may be replaced by their resultant $P$ determined according to Bradley's approach [26]. In this approach, the adhesive force between two unit areas is considered as a long-range Lennard-Jones force:

$$
\sigma(z)=\frac{8 \Delta \gamma}{3 \varepsilon}\left[\left(\frac{\varepsilon}{z}\right)^{3}-\left(\frac{\varepsilon}{z}\right)^{9}\right]
$$

Here, $\varepsilon$ is a phenomenological distance between two atoms/molecules, $z$ is the distance between two unit areas and $\Delta \gamma$ is Dupré adhesion energy defined by

$$
\Delta r=r_{1}+r_{2}-r_{12}
$$

where $\gamma_{1}$ and $\gamma_{2}$ are, respectively, the surface energies of the filaments, and $\gamma_{12}$ is the interface energy. For uniform filaments in contact, $\Delta \gamma=2 \gamma$. Thus, the adhesive force can be estimated by performing the integration with the integrant (15) such that

$$
\begin{aligned}
P= & \frac{8 \Delta \gamma}{3 \varepsilon} \int_{A} \sigma(z) \mathrm{d} A=\frac{8 \Delta \gamma}{3 \varepsilon} \int_{A}\left[\left(\frac{\varepsilon}{A x^{2}+B y^{2}-\alpha+h_{0}}\right)^{3}\right. \\
& \left.-\left(\frac{\varepsilon}{A x^{2}+B y^{2}-\alpha+h_{0}}\right)^{9}\right] \mathrm{d} A
\end{aligned}
$$

where the integration domain is over the region out of the contact zone on the tangential plane such that $A x^{2}+B y^{2} \geq$ $\alpha, h_{0}$ is the minimum gap between surfaces in contact. In Bradley's approach, it assumes $h_{0}=\varepsilon$. Therefore, substituting (5) into (17) leads to the resultant compressive force $P$ :

$$
P=\frac{4 \pi \gamma R}{\sin \varphi} \text {. }
$$

Interestingly, the adhesive force is independent of the size of the contact zone and equal to the adhesive force between two rigid uniform cylinders in contact [31].

Consequently, substitution of (5) and (18) into (14) yields the semi-axes of the elliptical contact zone as

$$
a=m \sqrt[3]{\frac{6 \pi\left(1-v^{2}\right)}{\sin \varphi} \frac{R^{2} \gamma}{E}}, \quad b=n \sqrt[3]{\frac{6 \pi\left(1-v^{2}\right)}{\sin \varphi} \frac{R^{2} \gamma}{E}}
$$

and $m$ and $n$ are functions with respect to $\varphi$ as tabulated in Table 1 [28]. The distance reduction of the filaments $\alpha$ due to adhesive forces can be determined by substituting (18) and (19) into (13):

$$
\alpha=\frac{6 \pi\left(1-v^{2}\right)}{\sin \varphi} \frac{\gamma R}{E} \int_{0}^{\infty} \frac{\mathrm{d} \xi}{\sqrt{\left(a^{2}+\xi\right)\left(b^{2}+\xi\right) \xi}} .
$$

Therefore, relations (18)-(20) govern the adhesive contact in filaments. It should be mentioned that additional compressive force may exist due to the coupling of surface tension and surface deformation. This force can be approximated as $\pi \mathrm{rab} / R$, a higher-order term compared with $R$ since $a b \ll R^{2}$ assumed in elastic contact theory. Furthermore, due to the viscoelastic properties of polymers, thin polymer fibers may creep near the contact zone under high adhesive compressive stress as observed in the experiment.

\section{Numerical evaluation and discussions}

Hereafter, we examine the variation of the area of contact zone between two fibers versus the fiber orientation angle $\varphi$ for several radii $R$ and intrinsic lengths of fiber material $r / E$. The area of the contact zone can be determined by relation (19) such that

$$
A_{c}=\pi a b=\pi m n\left[\frac{6 \pi\left(1-v^{2}\right)}{\sin \varphi} \frac{R^{2} \gamma}{E}\right]^{2 / 3} .
$$




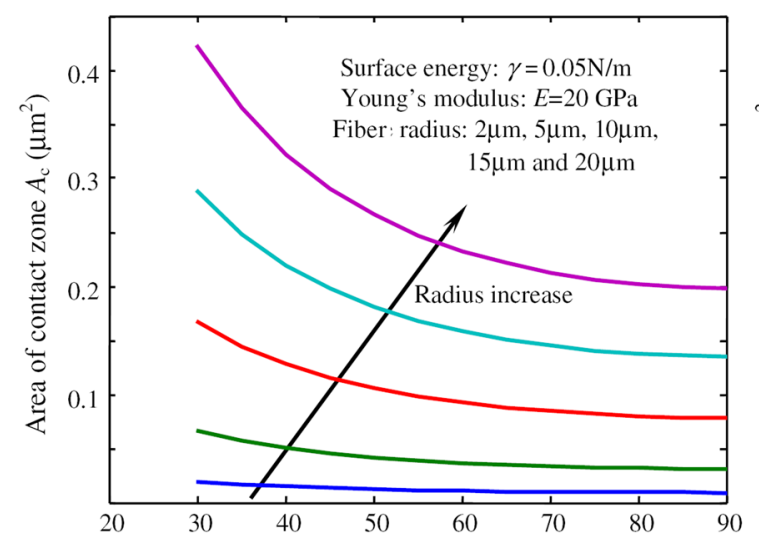

(a) Fiber orientation angle $\varphi\left(^{\circ}\right)$
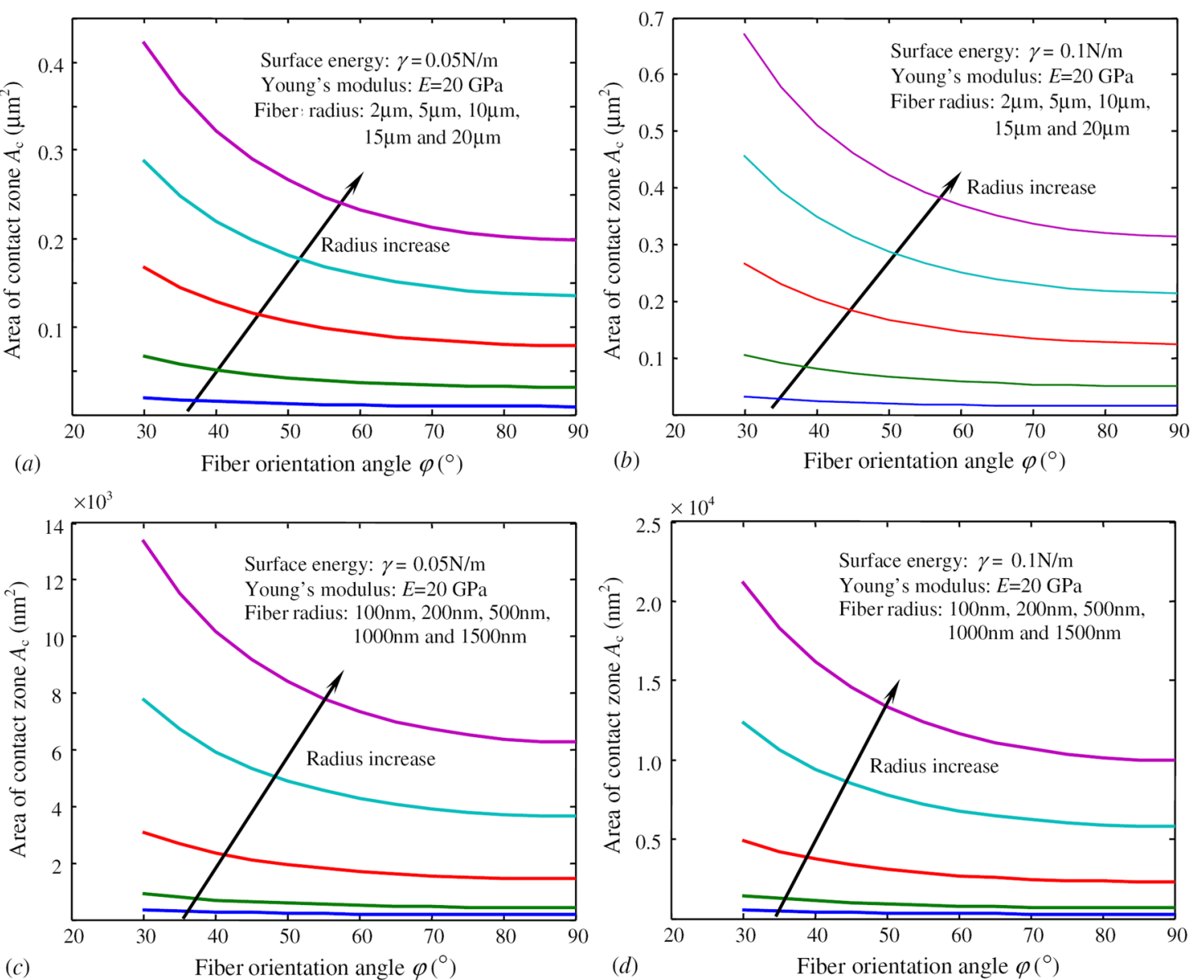

Figure 2. Variation of the area of contact zone versus the angle between fibers (a) $\gamma=0.05 \mathrm{Nm}^{-1}$ and (b) $\gamma=0.1 \mathrm{Nm}^{-1}$ for fiber radii of $2 \mu \mathrm{m}, 5 \mu \mathrm{m}, 10 \mu \mathrm{m}, 15 \mu \mathrm{m}$, and $20 \mu \mathrm{m}$, respectively and (c) $\gamma=0.05 \mathrm{Nm}^{-1}$ and (d) $\gamma=0.1 \mathrm{Nm}^{-1}$ for fiber radii of $100 \mathrm{~nm}, 200 \mathrm{~nm}$, $500 \mathrm{~nm}, 1 \mu \mathrm{m}$ and $1.5 \mu \mathrm{m}$, respectively.

During the numerical process, fiber surface energies are selected as $\gamma=0.05 \mathrm{Nm}^{-1}$ and $\gamma=0.1 \mathrm{Nm}^{-1}$, respectively, and Young's modulus is chosen as $E=20 \mathrm{GPa}$. These values are close to those of typical polymer fibers. Two groups of fibers are used. The first group has fiber radii of 2, 5, 10, 15, and $20 \mu \mathrm{m}$; and the second has fiber radii of 100, 200, 500, 1000 , and $1500 \mathrm{~nm}$. The corresponding numerical results are plotted in Figure 2. From Figure 2, it can be observed that for a given fiber radius, the area of the contact zone decreases rapidly with an increase of the angle $\varphi$ between fibers since the adhesive force decreases very fast with an increase in the angle $\varphi$. In the case of fibers in orthogonal contact, the area of the contact zone reaches its minimum value:

$$
\left(A_{c}\right)_{\min }=6^{2 / 3} \pi^{5 / 3}\left(1-v^{2}\right)^{2 / 3}\left(\frac{R^{2} \gamma}{E}\right)^{2 / 3} .
$$

In this case, the fiber pair has the minimum adhesive force based on relation (18), and therefore the fibers have the minimum deformation and elastic strain energy.

Relation (21) also indicates the scaling properties of the area of the contact zone with respect to the fiber radius and the intrinsic length, i.e.

$$
A_{\mathrm{c}} \propto R^{4 / 3} \quad \text { and } \quad A_{\mathrm{c}} \propto\left(\frac{\gamma}{E}\right)^{2 / 3} .
$$

Furthermore, it should be mentioned that for soft ultrathin fibers such as those electrospun polymer nanofibers with diameters around hundreds of nanometers and below, surface tension may lead to significant hydrostatic stress inside the nanofibers that is in the order of $\gamma / R(\sim 1$ $\mathrm{MPa}$ ). This stress may have an appreciable effect on the contact mechanics of nanofibers. For viscoelastic polymers, creeping may happen near the contact zone under high adhesive compressive stress that has been observed in experiment. As a first approach, we ignore these effects in the present study.

\section{Conclusions}

In this study, a simple 3D elastic contact model based on the DMT theory has been developed to examine the elastic contact in filaments induced by surface adhesion. Bradley's approach has been used successfully in determining the resultant compressive (adhesive) force, which is independent of the elastic deformation of the filaments in contact. Explicit solutions have been obtained for the compressive (adhesive) force, area of the contact zone and elastic deformation of the fibers. It shows that the compressive (adhesive) force and the area of the contact zone decrease rapidly with the increase of the angle between two filaments. In the case 
of orthogonal filaments in contact, the filaments have the minimum compressive (adhesive) force and the minimum area of contact zone. Corresponding numerical simulations have been performed to demonstrate the effects of filament radius and orientation, surface energies and elasticity on the elastic deformation and the size of the contact zone.

The present model is capable of determining the contact force, contact stress, and area of the contact zone. These parameters can be used in further exploring the mechanisms of filament friction, sliding, and compression hysteresis in fibrous materials subjected to external loading. Furthermore, for ultrathin soft fibers, the hydrostatic stress induced by surface tension and the material viscoelasticity may have an appreciable effect on the contact in filaments.

\section{Acknowledgment}

Partial support of this work by the NSF, AFOSR, and ARO/ ARL is gratefully acknowledged.

\section{References}

[1] Reneker D H and Chun I 1996 Nanotechnology 7216

[2] Dzenis Y 2004 Science 3041917

[3] Li D and Xia Y N 2004 Adv. Mater. 161151

[4] Kim J S and Reneker D H 1999 Polym. Compos. 20124

[5] Huang Z M, Zhang Y Z, Kotaki M, and Ramakrishna S 2003 Compos. Sci. Technol. 632223

[6] Chew S Y, Wen Y, Dzenis Y, and Leong K W 2006 Curr. Pharmaceutical Des. 124751

[7] Teo W E and Ramakrishna S 2006 Nanotechnology 17 R89

[8] Murugan R and Ramakrishna S 2006 Tissue Eng. 12435

[9] van Wyk C M 1946 J. Textile Inst. 37 T285

[10] Cox H L 1952 Br. J. Appl. Phys. 372
[11] Narter M A, Batra S K, and Buchanan D R 1999 Proc. R. Soc. Lond. A 4553543

[12] Carnaby G A and Pan N 1989 Textile Res. J. 59275

[13] Pan N and Carnaby G A 1989 Textile Res. J. 59285

[14] Toll S and Manson J A E 1995 J. Appl. Mech. - Trans. ASME. 62223

[15] Astrom J A, Makinen J P, Alava M J, and Timonen J 2000 Phys. Rev. E 615550

[16] Astrom J A, Makinen J P, Hirvonen H, and Timonen J 2000 J. Appl. Phys. 885056

[17] Wang C W, Berhan L, and Sastry A M 2000 J. Eng. Mater. Technol. - Trans. ASME 122450

[18] Wang C W and Sastry A M 2000 J. Eng. Mater. Technol. Trans. ASME 122460

[19] Sastry A M, Wang C W, and Berhan L 2001 Key Eng. Mater. 200229

[20] Wu X F and Dzenis Y A 2005 J. Appl. Phys. 98093501

[21] Chatterjee A P 2006 J. Appl. Phys. 100054302

[22] Chatterjee A P and Prokhorova D A 2007 J. Appl. Phys. 101 104301

[23] Wu X F and Dzenis Y A 2006 J. Appl. Phys. 100124318

[24] Wu X F and Dzenis Y A 2007 Nanotechnology 18285702

[25] Derjaguin B V, Muller V M, and Toporov Y P 1975 J. Colloid Interface Sci. 53314

[26] Bradley R S 1932 Phil. Mag. 13853

[27] Johnson K L 1985 Contact Mechanics (Cambridge: Cambridge University Press)

[28] Timoshenko S and Goodier J N Theory of Elasticity 2nd edition (New York: McGraw-Hill)

[29] Landau L D and Lifshitz E M 1986 Theory of Elasticity 3rd edition (London: Butterworth-Heinemann)

[30] Maugis D 2000 Contact, Adhesion and Rupture of Elastic Solids (Heidelberg: Springer)

[31] Israelachvili J 1992 Intermolecular and Surface Forces 2nd edition (New York: Academic) 